Original Article

\title{
Validity and reliability of isometric muscle strength measurements of hip abduction and abduction with external hip rotation in a bent-hip position using a handheld dynamometer with a belt
}

\author{
Hidefumi Aramaki, RPT, MS ${ }^{1)^{*}}$, Munenori Katoh, RPT, PhD ${ }^{1)}$, Yukinobu Hitragi, RPT, PhD ${ }^{1)}$, \\ Tsubasa Kawasaki, RPT, PhD ${ }^{1)}$, Tomohisa Kurihara, RPT ${ }^{2)}$, Yorikatsu Ohmi, RPT, MS ${ }^{2)}$ \\ 1) Department of Physical Therapy, Faculty of Health Sciences, Ryotokuji University: 5-8-1 Akemi, \\ Urayasu-shi, Chiba 279-8567, Japan \\ 2) Department of Rehabilitation, Nippon Koukan Hospital, Japan
}

\begin{abstract}
Purpose] This study aimed to investigate the relatedness, reliability, and validity of isometric muscle strength measurements of hip abduction and abduction with an external hip rotation in a bent-hip position using a handheld dynamometer with a belt. [Subjects and Methods] Twenty healthy young adults, with a mean age of $21.5 \pm$ 0.6 years were included. Isometric hip muscle strength in the subjects' right legs was measured under two posture positions using two devices: a handheld dynamometer with a belt and an isokinetic dynamometer. Reliability was evaluated using an intra-class correlation coefficient (ICC); relatedness and validity were evaluated using Pearson's product moment correlation coefficient. Differences in measurements of devices were assessed by two-way ANOVA. [Results] ICC $(1,1)$ was $\geq 0.9$; significant positive correlations in measurements were found between the two devices under both conditions. No main effect was found between the measurement values. [Conclusion] Our findings revealed that there was relatedness, reliability, and validity of this method for isometric muscle strength measurements using a handheld dynamometer with a belt.

Key words: Handheld dynamometer with a belt, Isometric muscle strength measurements, Validity and reliability
\end{abstract}

(This article was submitted Feb. 19, 2016, and was accepted Apr. 7, 2016)

\section{INTRODUCTION}

A handheld dynamometer (HHD) is a device used to measure muscular strength using a ratio scale of measurement. In the conventional measuring method using an HHD, the measurer holds the HHD in a hand and then measures muscle strength. However, this method has a limitation regarding fixation of the $\mathrm{HHD}^{1,2)}$. As a countermeasure for this problem, measurement methods in combination with a stick ${ }^{3)}$, steel support ${ }^{4}$, or belt ${ }^{5,6)}$ have been reported to date.

Katoh et al. ${ }^{7)}$ measured lower limb muscle strength using a method for fixing an HHD with a belt and examined its validity by comparing the measured values with those of an isokinetic dynamometer (IKD). Their results demonstrated that the Pearson's correlation coefficients between the measurement methods were as follows hip flexion, $\mathrm{r}=0.52$; hip extension, $\mathrm{r}=0.84$; hip abduction (ABD), $\mathrm{r}=0.34(<450 \mathrm{~N} ; \mathrm{r}=0.65)$; hip adduction, $\mathrm{r}=0.52$; hip external rotation (ER), $\mathrm{r}=0.86$; hip internal rotation, $\mathrm{r}=0.77$; knee flexion, $\mathrm{r}=0.88$; and knee extension, $\mathrm{r}=0.75$. From these results, use of an HHD fixed with a belt was concluded to be a valid strength measurement method, except in subjects who have a high level of hip abductor strength. However, as the correlation coefficient for hip $\mathrm{ABD}$ was the lowest, it was also considered to be necessary to revise the

\footnotetext{
*Corresponding author. Hidefumi Aramaki (E-mail: aramaki@ryotokuji-u.ac.jp)

(C)2016 The Society of Physical Therapy Science. Published by IPEC Inc.

This is an open-access article distributed under the terms of the Creative Commons Attribution Non-Commercial No Derivatives (by-nc-nd) License $<$ http://creativecommons.org/licenses/by-nc-nd/4.0/>.
} 
measurement method.

$\mathrm{ABD}$ with hip ER in the hook-lying position is called the bent knee fall out (BKFO) ${ }^{8}$. Moreover, ABD with ER in hip and knee flexion positions on side-lying is called the clamshell (CLAM) exercise ${ }^{9-12)}$. The BKFO and CLAM are used as hip joint exercises in a non-load environment. Electromyography activities of the gluteus medius during performance of the CLAM are lower than those during crotch ABD exercises conducted in the hip and knee extension positions ${ }^{9,10)}$. Moreover, the authors reported that the activity of the gluteus medius during the CLAM is lower than that during hip ABD exercises conducted in the hip and knee extension positions. Thus, these findings suggest that the activity of the gluteus medius is changed by the hip flexion angle ${ }^{9,10)}$. However, the activities of the tensor fascia latae, gluteus maximus, and anterior hip flexors are not significantly different even if the flexion angle of the hip joint is changed ${ }^{11)}$. Therefore, the CLAM is used as a movement to lower the load of the gluteus medius.

When the CLAM and BKFO are used as hip-strengthening exercises, it is necessary to measure the muscle strength to set the amount of load and to achieve the appropriate effects. However, a search in PubMed on December 20, 2015, using the key words "muscle strength," "clamshell," "muscle strength," and "bent knee fall out" did not find any reports. Clarifying the reliability and validity of muscle strength measurements in the CLAM and BKFO positions would contribute to design of effective exercises.

Therefore, this study aimed to examine the reliability and validity of muscular strength measurements in CLAM, BKFO, and revised $\mathrm{ABD}$ muscular strength measurement. In addition, this study aimed to examine the correlation among the muscular strength measurements.

\section{SUBJECTS AND METHODS}

The subjects comprised 20 young healthy volunteers (nine females) with no history of orthopedic disease in the limbs or trunk of the body or cardiac disease. The mean age was $21.5 \pm 0.6$ years, mean height was $164.3 \pm 9.4 \mathrm{~cm}$, and mean weight was $59.3 \pm 14.0 \mathrm{~kg}$. The study aim and details were explained to the subjects, and measurements were performed after obtaining informed written consent. Approval from the Human Ethics Committee of Ryotokuji University (approval number 2537) was obtained for this study, and it adhered to the tenets of the Declaration of Helsinki.

The isometric hip joint and muscular strengths of the right leg were measured using an HHD and IKD, respectively. The assessor was a male physiotherapist (height, $167.0 \mathrm{~cm}$; weight, $69.0 \mathrm{~kg}$; and 18 years of experience). For blinding of assessors and subjects, an assistant performed the reading and recording of measurement values.

Muscle strength was measured under two conditions: 1) ABD and ER with hip flexion and 2) ABD. The ABD and ER muscle strengths in the hip joint were measured using an HHD in the supine position (HHD-BKFO), using an HHD in the side-lying position (HHD-CLAM), and using an IKD in the side-lying position (IKD-CLAM). ABD and ER with hip flexion were performed with hip flexion of $45^{\circ}$ and knee flexion of $90^{\circ}$ (Fig. 1). ABD was measured using an HHD in the supine position (HHD-ABD), using an HHD in the side-lying position (HHD-SL ABD), and using an IKD in the side-lying position (IKD-SL ABD). In the case of measurement in the side-lying position, the strength of the upper side of the lower limb was measured (Fig. 2). The subjects inserted a cushion between both the lower limbs to achieve measurement in a neutral adduction position /ABD in the hip joint.

A $\mu$ TAS F-1 (Anima Corp, Tokyo, Japan) was used as the HHD for measuring muscle strength. An HHD sensor was fixed in place using a belt, and the isometric muscular strength was measured. The location for applying the HHD sensor (the location of the distal part of the sensor), belt location, joint angles, and fixed location at the time of measurement are shown in Table 1. The measurer maintained the direction of the sensor during measurement. Following a single practice trial, the subjects performed isometric contraction at their maximum exertion for approximately $5 \mathrm{~s}$, and the maximum muscle strength during this task was recorded. A second measurement was performed after at least $30 \mathrm{~s}$ of rest.

A Biodex System 3 (Biodex Medical Systems, Shirley, NY, USA) was used as the IKD equipment. The IKD pad position (the position of the distal part of the pad) was the same as the HHD sensor position (Table 1). In the IKD-CLAM measurement, the seat was rotated $45^{\circ}$, and a stand, which was the same height as the seat, was set to prevent falling of the leg. Moreover, both legs were fixed with a strap to prevent deviation in the external hip rotation axis (Fig. 1). Following a single practice trial, the subjects performed three trials of isometric maximum voluntary contraction for approximately $5 \mathrm{~s}$ with rests of at least $30 \mathrm{~s}$ between each, and the peak torque value was recorded.

The order of HHD and IKD measurements was counterbalanced. The orders of the four measurements using the HHD and the two measurements using the IKD were also counterbalanced. There was at least 5 min of rest between measurements with different settings.

The unit of measurement for the HHD was $\mathrm{kg}$. Therefore, $\mathrm{Nm}\left(\mathrm{kg}, \mathrm{m} / \mathrm{s}^{2}\right)$ was calculated based on the acceleration of gravity $\left(9.8 \mathrm{~m} / \mathrm{s}^{2}\right)$ and the lever arm length (distance from the greater trochanter to the sensor center, $\mathrm{m}$ ) to perform the comparison with the IKD measurement values.

The intra-class correlation coefficient (ICC) was used to evaluate the intra-rater reliability of the measurement values using the HHD. The validity of the measurement values using the HHD was investigated using Pearson's product moment correlation coefficient with respect to the standard rated validity and by setting the IKD measurement values as the external standard. 
The differences in measurement values were assessed using two-way repeated measures analysis of variance (ANOVA) with the measurement device [HHD (supine), HHD (SL), and IKD (SL)] and measurement posture (ABD and ER in hip flexion, $\mathrm{ABD}$ ) as the two factors; the two highest measurement values were used for this. Moreover, the ratio of the HHD measurement value to the IKD measurement value (HHD/IKD; the HHD/IKD ratio) was obtained.

All statistical analyses were performed using the R2.8.1 statistical software, and $p$ values of $<0.05$ were deemed significant.

\section{RESULTS}

The ICC $(1,1)$ for the values of two consecutive measurements using the HHD was 0.94-0.98 (Table 2). For each movement item, the Pearson's correlation coefficient between the HHD in the supine position and IKD and between the HHD in the side-lying position and IKD were $r=0.82-0.86$; significantly positive correlations were found in both cases. In all measurement methods, the Pearson's correlation coefficient between the two types of movement were $\mathrm{r}=0.86-0.88$, and significantly positive correlations were found for all. Also, there were significant positive correlations between the other

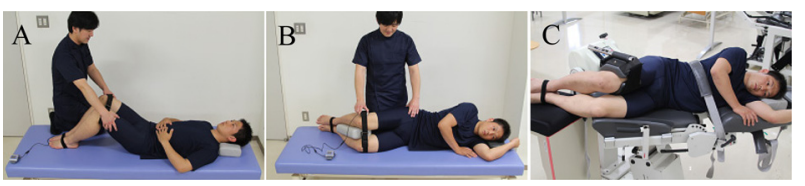

Fig. 1. Hip abduction and external rotation with hip flexion. A, handheld dynamometer, bent knee fall out; B, handheld dynamometer, clamshell; $\mathrm{C}$, isokinetic dynamometer, clamshell

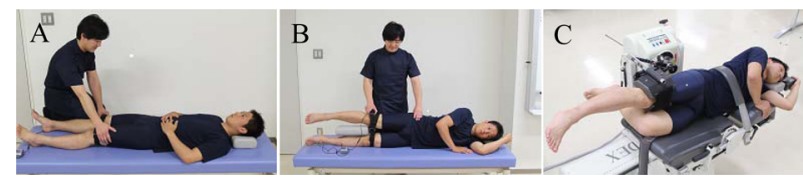

Fig. 2. Hip abduction. A, handheld dynamometer, hip abduction; $\mathrm{B}$, handheld dynamometer, side-lying hip abduction; $\mathrm{C}$, isokinetic dynamometer, side-lying hip abduction

Table 1. Measurement methods

\begin{tabular}{|c|c|c|c|c|}
\hline Abbreviation & Exercise & Posture & Hip $^{\text {a) }}$ & Knee joint \\
\hline HHD-ABD & Hip abduction & Supine & Middle & Extension \\
\hline HHD-SL ABD & Hip abduction & Side-lying & Middle & Extension $^{\mathrm{b})}$ \\
\hline IKD-SL ABD & Hip abduction & Side-lying & Middle & Extension \\
\hline HHD-BKFO & Hip abduction and external rotation & Supine & $45^{\circ}$ flexion & $90^{\circ}$ flexion \\
\hline HHD-CLAM & Hip abduction and external rotation & Side-lying & $45^{\circ}$ flexion & $90^{\circ}$ flexion $^{\mathrm{b})}$ \\
\hline IKD-CLAM & Hip abduction and external rotation & Side-lying ${ }^{\text {a) }}$ & $45^{\circ}$ flexion & $90^{\circ}$ flexion \\
\hline Abbreviation & Sensor pad location & Belt location & \multicolumn{2}{|c|}{ Fixed location } \\
\hline HHD-ABD & Upper lateral thigh bone $^{c}$ & Both thighs & \multicolumn{2}{|c|}{ Thigh (non-measurement side) } \\
\hline HHD-SL ABD & Upper lateral thigh bone ${ }^{c}$ & Both thighs & \multicolumn{2}{|c|}{ Pelvis (measurement side) } \\
\hline IKD-SL ABD & Straight on the lateral thigh bone & - & \multicolumn{2}{|c|}{ Thigh/trunk (measurement side) } \\
\hline HHD-BKFO & Upper lateral thigh bone ${ }^{c)}$ & Both thighs & \multicolumn{2}{|c|}{ Thigh/leg (measurement side) } \\
\hline HHD-CLAM & Upper lateral thigh bone ${ }^{\mathrm{c}}$ & Both thighs & \multicolumn{2}{|c|}{ Pelvis/leg (measurement side) } \\
\hline IKD-CLAM & Straight on the lateral thigh bone & - & \multicolumn{2}{|c|}{ Thigh/trunk/ankles (Measurement side) } \\
\hline
\end{tabular}

HHD: handheld dynamometer; ABD: hip abduction; SL: side-lying; IKD: isokinetic dynamometer; BKFO: bent knee fall out; CLAM: clamshell.

a) Lower extremity supported on same plain, b) Cushion placed between legs, ${ }^{c}$ Sensor held in correct orientation

Table 2. HHD measurement test-retest reliability

\begin{tabular}{lcc}
\hline & ICC $(1,1)$ & $95 \%$ CI \\
\hline ABD & 0.98 & $0.94-0.99$ \\
SL ABD & 0.94 & $0.86-0.98$ \\
BKFO & 0.98 & $0.95-0.99$ \\
CLAM & 0.97 & $0.94-0.99$ \\
\hline
\end{tabular}

HHD: handheld dynamometer; ABD: hip abduction; SL: side-lying; BKFO: bent knee fall out; CLAM: clamshell; ICC: intraclass correlation coefficient; CI: confidence interval 
measurement values (Table 3).

The values obtained from measurements using the HHD and IKD are presented in Table 4. The results of two-way repeated measures ANOVA did not determine main effects for the measurement device, measurement posture, movement type, or interaction between factors. The HHD/IKD ratio was 0.87-0.97 (Table 4).

\section{DISCUSSION}

The results showed that the $\operatorname{ICC}(1,1)$ for the values obtained from measurements using the HHD was $\geq 0.9$, and the reliability was considered to be high. Previously, Katoh et al. ${ }^{13)}$ reported reliability amongst assessors in hip ABD muscle strength measurement using an HHD and a belt. Their results from performing three measurement sessions, one in the morning, afternoon of the same day, and 1 week later, showed that the ICC $(1,1)$ for two consecutive measurements of hip ABD ranged between 0.93 and 0.95 . In previous studies, the HHD/IKD ratio was 0.34 for hip ABD, and a significantly lower measurement value was obtained with an HHD compared with an $\mathrm{IKD}^{7}$. The results of the present study revealed that the ICC $(1,1)$ was $0.94-0.98$, which was similar to the high values obtained in previous studies. However, the results of this study did not reveal significant differences in the mean values, and the HHD/IKD ratio was close to 1. Moreover, because there was significant correlation between the HHD and IKD measurement values, it was considered that the ABD measurement method utilized a superior fixation method compared with the methods used in proceeding studies and thus provided higher validity. Because the lower limbs on the non-measurement side were used for belt fixation in this measurement method, it was expected that the muscular strength value on the measurement side decreased when there was hip extension muscle weakness on the non-measurement side. It was considered that examination of the method for measurement of that case is necessary.

There was no significant difference between the mean values for CLAM muscle strength using the HHD and IKD, and high correlation was found. Therefore, it can be considered that the validity of CLAM muscle strength measurement using the HHD was high.

There was no significant difference between CLAM muscle strength using the HHD or IKD and the BKFO muscle strength, and high correlation was demonstrated. This suggests that the BKFO can be used as a method that is interchangeable with the CLAM in patients who have difficulty in side-lying.

Furthermore, there was a significant correlation between CLAM and ABD measurement values. Significant positive correlations between the other measurement values were also found, which was acceptable because the same agonist muscle was used for these movements. Therefore, it was considered that CLAM measurement values should be used in place of hip ABD muscle strength measurement values in patients who have difficulty with hip or knee extension.

The limitation of this study was that the subjects were healthy young people, and it was unclear whether similar results would be obtained in elderly people who are frail or suffering from muscle- or bone-related diseases. Therefore, it is desirable to perform further investigations on different subjects.

Table 3. Correlations among measurements

\begin{tabular}{lcccccc}
\hline & HHD-ABD & HHD-SL ABD & IKD-SL ABD & HHD-BKFO & HHD-CLAM & IKD-CLAM \\
\hline HHD-ABD & 1 & $0.85^{* *}$ & $0.82^{* *}$ & $0.88^{* *}$ & $0.82^{* *}$ & $0.73^{* *}$ \\
HHD-SL ABD & & 1 & $0.82^{* *}$ & $0.92^{* *}$ & $0.86^{* *}$ & $0.77^{* *}$ \\
IKD-SL ABD & & & 1 & $0.89^{* *}$ & $0.78^{* *}$ & $0.87^{* *}$ \\
HHD-BKFO & & & & 1 & $0.93^{* *}$ & $0.86^{* *}$ \\
HHD-CLAM & & & & & 1 & $0.84^{* *}$ \\
IKD-CLAM & & & & & & 1 \\
\hline
\end{tabular}

HHD: handheld dynamometer; ABD: hip abduction; SL: side-lying; IKD: isokinetic dynamometer; BKFO: bent knee fall out, CLAM: clamshell. ${ }^{* *} \mathrm{p}<0.01$

Table 4. Average values of strength measurements

\begin{tabular}{lccccc}
\hline & HHD & HHD & IKD & \multicolumn{2}{c}{ HHD/IKD ratio } \\
\cline { 5 - 5 } & Supine & Side-lying & Side-lying & Supine & Side-lying \\
\hline Hip abduction & 99.8 & 98.1 & 103.2 & 0.97 & 0.95 \\
& $(\mathrm{SD}=29.4)$ & $(\mathrm{SD}=33.2)$ & $(\mathrm{SD}=34.1)$ & & \\
Hip abduction and external rotation & $91.9^{\text {a) }}$ & $90.0^{\mathrm{b})}$ & $103.9^{\mathrm{b})}$ & $0.88^{\mathrm{a})}$ & $0.87^{\mathrm{b})}$ \\
with hip flexion & $(\mathrm{SD}=31.4)$ & $(\mathrm{SD}=36.8)$ & $(\mathrm{SD}=41.5)$ & & \\
\hline $\begin{array}{l}\text { Mean }(\mathrm{SD}) \\
\text { clamshell }\end{array}$ & &
\end{tabular}


In conclusion, it was considered that $\mathrm{ABD}$ and $\mathrm{ER}$ (CLAM, BKFO) muscle strength measurement and the revised ABD muscle strength measurement in a bent-hip position using an HHD together with belt fixation, have high reliability and validity. Furthermore, there was a significant correlation between each muscular strength measurement, and it was considered that CLAM measurement values should be used in place of hip ABD muscle strength measurement values in patients who have difficulty with hip or knee extension.

\section{REFERENCES}

1) Hyde SA, Goddard CM, Scott OM: The myometer: the development of a clinical tool. Physiotherapy, 1983, 69: 424-427. [Medline]

2) Wiles CM, Karni Y: The measurement of muscle strength in patients with peripheral neuromuscular disorders. J Neurol Neurosurg Psychiatry, 1983, 46: 1006-1013. [Medline] [CrossRef]

3) Johansson KM, Adolfsson LE: Intraobserver and interobserver reliability for the strength test in the Constant-Murley shoulder assessment. J Shoulder Elbow Surg, 2005, 14: 273-278. [Medline] [CrossRef]

4) Brinkmann JR: Comparison of a hand-held and fixed dynamometer in measuring strength of patients with neuromuscular disease. J Orthop Sports Phys Ther, 1994, 19: 100-104. [Medline] [CrossRef]

5) Katoh M, Yamasaki H: Comparison of reliability of isometric leg muscle strength measurements made using a hand-held dynamometer with and without a restraining belt. J Phys Ther Sci, 2009, 21: 37-42. [CrossRef]

6) Katoh M: Reliability of isometric shoulder muscle strength measurements made by a hand-held dynamometer with a belt. J Phys Ther Sci, 2015 , 27: 851-854. [Medline] [CrossRef]

7) Katoh M, Hiiragi Y: Validity of isometric muscle strength measurements of the lower limbs using a hand-held dynamometer and belt: a comparison with an isokinetic dynamometer. J Phys Ther Sci, 2011, 23: 553-557. [CrossRef]

8) Paul DJ, Nassis GP, Whiteley R, et al.: Acute responses of soccer match play on hip strength and flexibility measures: potential measure of injury risk. J Sports Sci, 2014, 32: 1318-1323. [Medline] [CrossRef]

9) Distefano LJ, Blackburn JT, Marshall SW, et al.: Gluteal muscle activation during common therapeutic exercises. J Orthop Sports Phys Ther, 2009, 39: 532-540. [Medline] [CrossRef]

10) Sidorkewicz N, Cambridge ED, McGill SM: Examining the effects of altering hip orientation on gluteus medius and tensor fascae latae interplay during common non-weight-bearing hip rehabilitation exercises. Clin Biomech (Bristol, Avon), 2014, 29: 971-976. [Medline] [CrossRef]

11) McBeth JM, Earl-Boehm JE, Cobb SC, et al.: Hip muscle activity during 3 side-lying hip-strengthening exercises in distance runners. J Athl Train, 2012, 47: 15-23. [Medline]

12) Boren K, Conrey C, Le Coguic J, et al.: Electromyographic analysis of gluteus medius and gluteus maximus during rehabilitation exercises. Int J Sports Phys Ther, 2011, 6: 206-223. [Medline]

13) Katoh M, Yamasaki H: Test-retest reliability of isometric leg muscle strength measurements made using a hand-held dynamometer restrained by a belt: comparisons during and between sessions. J Phys Ther Sci, 2009, 21: 239-243. [CrossRef] 